Open Access

\title{
On the performance of an enhanced transmission scheme for cooperative relay networks with NOMA
}

\author{
Wei Duan ${ }^{1}$, Guoan Zhang ${ }^{1}$, Qiang Sun ${ }^{1,4}$, Jia Hou ${ }^{2 *}$, Yancheng Ji ${ }^{1}$ and Jeaho Choi ${ }^{3}$
}

\begin{abstract}
In this paper, an enhanced transmission scheme for cooperative relaying networks with non-orthogonal multiple access (ECRN-NOMA) is proposed. In the proposed scheme, two different kinds of transmission schemes are investigated which are the single signal transmission and the enhanced superposition transmission schemes. Particularly, for the single transmission scheme, a successive interference cancellation (SIC) is utilized to decode the received signal, sent by the relay, at the destination. On the other hand, for the enhanced superposition coded signal transmission scheme, a maximum ratio combining (MRC) is utilized at the destination to improve the ergodic sum-rate (SR) of the proposed system. Specifically, two power allocation (PA) strategies are comprehensively discussed to characterize the performance of our proposed scheme. The achievable average SR of the proposed systems are analyzed for independent Rayleigh fading channels, and also their asymptotic expressions are also provided. Qualitative numerical results corroborating our theoretical analysis show that the enhanced superposition coded signal transmission scheme applied to the proposed ECRN-NOMA improves the SR performance significantly in comparison to the others.
\end{abstract}

Keywords: Non-orthogonal multiple access, Ergodic sum-rate, Maximum ratio combining power, Allocation

\section{Introduction}

A non-orthogonal multiple access (NOMA) technique has been widely considered as a promising multiple access (MA) potential candidate for future wireless networks due to its superior spectral efficiency $[1,2]$. Different from the traditional orthogonal multiple access (OMA) techniques $[3,4]$ such as frequency-division multiple access (FDMA) and time-division multiple access (TDMA), NOMA explores the non-orthogonal resource allocation. The key idea of NOMA is to explore the power domain for realizing MA, where different users are served at different power levels [5]. In NOMA, signals of multiple users are superimposed in the power domain at the transmitter while the successive interference cancellation (SIC) is conducted on the received superimposed signal at the receiver, respectively. As composing a superimposed signal for transmission, an uneven power allocation can

*Correspondence: houjia@suda.edu.cn

${ }^{2}$ School of Electronics and Information, Soochow University, Suzhou, China Full list of author information is available at the end of the article be used in general so that a receiver with a lower channel gain is assigned with a higher power and a receiver with a higher channel gain with a lower power. Furthermore, the authors in [6] provided an overview of the latest NOMA research and innovations as well as their applications. Since multiple users can be served simultaneously, it is also shown that NOMA networks also reduce the delay since users are no longer forced to wait until an orthogonal resource block becomes available. A comprehensive overview of the present and emerging powerdomain SC-based NOMA research into 5G is studied in [7], where it also offers a general view of some implementation issues, including computational complexity, error propagation, deployment environments, and standardization status. In addition, a resource allocation for downlink non-orthogonal multiple access systems is proposed in [8], in which, the former pairs the users to obtain the high capacity gain, while the latter allocates power to users to balance system throughput and user fairness. Furthermore, the authors in [9] investigated a dual-hop cooperative relaying scheme using NOMA, where two sources 
communicate with their corresponding destinations via a common relay.

Conventionally, due to the compatibility of the NOMA technology with other communication technologies, it can be integrated in existing and future wireless systems. In [10-13], the secrecy issue of NOMA has been considered, which improves the security capability, the reliability and the transmission rate in the physical layer communication. In [14], the authors have proposed a complete resource allocation-based user selection scheme which has a low computational complexity with excellent performance for both perfect and imperfect channel state information (CSI) scenarios. Moreover, a best-near best-far user selection scheme is proposed in [15]. The application of cooperative simultaneous wireless information and power transfer (SWIPT) to NOMA networks are investigated in [15-18], where the NOMA-strong users are considered as energy harvesting relays to help the NOMA-weak users. It is worth noting that, on top of their spectral efficiency and outage performance, the fairness is also an important issue in NOMA systems [19, 20], since there is a trade-off between the total throughput and the user fairness. In addition, since index modulation (IM) [21-26] technology has the superiorities for the energy efficiency and low complexity, the authors investigated NOMA-based IM to mitigate inter-user interference, while maintaining high spectral efficiency [27, 28].

Moreover, multiple-input multiple-output (MIMO) [29-31] systems have been widely considered as a candidate for the fifth generation (5G) wireless communication due to their transmission reliability. The authors in [32] proposed cooperative NOMA systems in MIMO channels, which maximizes the achievable rate from the base station to the cell-edge user under transmit power constraints and achievable rate constraint from the base station to the central user. Considering the imperfect channel state information (CSI), a robust beamforming design is investigated for NOMA systems in MIMO channels [33]. Specifically, the joint power allocation and relay beamforming design for a NOMA amplify-and-forward (AF) relay network is studied in [34], where an alternating optimization-based algorithm is proposed to maximize the achievable rate of the destination.

Recently, the cooperative relay networks (CRNs) are drawing much attention because the relaying transmission is a promising technique which can be applied to increase the system capacity. Currently, the CRN-NOMA systems are widely studied [1, 15, 16, 18, 35-41]. In [35], a NOMA-based cooperative relaying system over Rician fading channels is studied and an analytical framework is developed to evaluate its performance. In [36], an accurate closed form approximation is obtained for the exact outage probability of a CRN-NOMA with an amplifyand-forward (AF) relay. Specifically, in [37], the resource allocation problem is studied for a NOMA wireless network with a one-way orthogonal frequency division multiplexing (OFDM) AF relay in order to optimize subchannel assignment and power allocation. In addition, the work of the NOMA-based cooperative AF relaying strategy over Nakagami-m fading channels is introduced, and the authors show that the cooperative NOMA outperforms conventional OMA systems [38]. A cooperative NOMA transmission technique using MRC is studied in [1]. This technique exploits prior information in NOMA systems. Moreover, the work of NOMA in the coordinated direct and relay transmission (CDRT) with the decodeand-forward (DF) protocol has been introduced in [39], it includes exact and asymptotic expressions for achievable rates of the system driven in independent Rayleigh fading channels. Since the performance of the achievable rate is limited by a poor channel, a novel receiver design for the CRN-based NOMA by using MRC is proposed in [40], which has advantages in the view point of ergodic SR and outage probability. Unfortunately, the proposed scheme in [40] requires a symbol allocated with a lower power to be decoded first, which degrades the outage performance. In order to further improve the outage performance, a two-stage power allocation CRS using NOMA is proposed in [41], in which the relay nodes forward a new superposition-coded symbol with a different power allocation.

Consider a superposition-coded signal is transmitted to the relays. In our proposed system, there are three kinds of transmissions, namely, CTRS-NOMA superposition case, CTRS-NOMA single case I, and CTRS-NOMA single case II, respectively. For the CTRS-NOMA superposition case, both of two relay nodes forward the superposition coded signal $x_{R}$ to the destination. Without loss of generality, for the CTRS-NOMA single case, we discussed two kinds of the power allocation schemes, i.e., for relay nodes $i$, the transmitted symbol $x_{i}$ is allocated with a higher PA factor for case I, while a lower PA factor for case II. Therefore, in this manner, the proposed CTRS-NOMA single case contains all the possible transmission strategies, which is necessary to employ two relays to support that of the strategies. In addition, the proposed scheme is possible to provide the mentality to study the multi-relaying systems and applicable in the future cooperative NOMA networks. These motivate us to investigate an enhanced transmission scheme with two relays. The implementations and contributions of this paper are summarized in the following:

- In order to further improve the ergodic SR, we comprehensively investigate an enhanced 2-stage superposed transmission scheme for CRN-NOMA with two relays is considered, where, unlike existing works, not only the source but also the relays are 
allowed to transmit superposition-coded signals, which is more general and challenging.

- Without loss of the generality, in the proposed system, two different kinds of transmission schemes are proposed. Specifically, the first scheme is named CTRS-NOMA single case, which is similar to the single relay scenario. Upon a reception from the source, each relay decodes-and-forwards a single corresponding symbol to the destination. Assuming that the CSI is perfectly known at the destination, the received signals can be decoded by utilizing SIC. In addition, two kinds of PA methods are analyzed. Specifically, the signal with more power at the source is allocated with a lesser power at the relay, while the other signal with lesser power at the source is allocated with a more power at the relay. Otherwise, an opposite PA scheme is considered. On the other hand, the second scheme is named CTRS-NOMA superposition case; unlike the existing works, not only source but also all relay nodes are allowed to forward superposition-coded signals to the destination. Upon a signal reception, MRC is utilized to maximize the system performance.

- Closed-form solutions of the ergodic SR for all the transmission schemes at high transmit signal-to-noise ratio (SNR) are derived with a negligible performance loss. It is worth noting that, there is few works that focus on the 2-stage superposed transmission for multiple relay networks, since it is hard to obtain the exact expression of the ergodic SR. Furthermore, the theoretical results are shown to highly agree with the simulation results, especially in the high SNR region.

- Through the numerical results, both analytically and numerically, we compare the proposed NOMA schemes with the TDMA scheme in terms of ergodic SR. It is shown that, the proposed CTRS-NOMA superposition case outperforms the TDMA and other two NOMA schemes significantly.

The rest of the paper is organized as follows. Section 2 describes a system model of the CTRS-NOMA and two proposed transmission schemes. In Section 3, the performance of two proposed systems in terms of achievable ergodic SRs are analyzed, and the numerical results are presented in order to corroborate the performance of two schemes of our CTRS-NOMA system in Section 4. Finally, Section 5 concludes this paper.

\section{System model}

Consider a simple CTRS consisting of one source, two relays, and one destination, and assume all nodes operate in a half-duplex mode, where the decode-and-forward (DF) scheme is considered, as shown in Fig. 1. The channels from the source to the relay node $i \in\{1,2\}$ and from the relay node $i$ to the destination are denoted as $h_{S R_{i}}$ and $h_{R_{i} D}$, respectively, and they are assumed to be independent complex Gaussian random variables with variances $\alpha_{S_{i} R}$, and $\alpha_{R_{i} D}$, respectively. In our proposed scheme, each transmission involves two time slots. Note that according to the NOMA principle, the power allocation factors $a_{1}$ and $a_{2}$ are with $a_{1}>a_{2}$ and $a_{1}+a_{2}=1$, which are related to the quality of the channel coefficients. At the first time slot, assuming the adoption of the superposition code, the superposed signal

$$
\sqrt{a_{1} P_{t}} x_{1}+\sqrt{a_{2} P_{t}} x_{2}
$$

is transmitted from the source to the relay node $i$, where $x_{i}$ denotes the broadcasted symbol at the source, and $P_{t}$ stands for the total transmit power.

The received signal at the relay node $i$ is given by

$$
y_{R_{i}}=h_{S R_{i}}\left(\sqrt{a_{1} P_{t}} x_{1}+\sqrt{a_{2} P_{t}} x_{2}\right)+n_{R_{i}}
$$

where $n_{R_{i}} \sim \mathcal{C N}\left(0, \sigma^{2}\right)$ denotes the additive white Gaussian noise (AWGN) with zero mean and variance $\sigma_{R_{i}}^{2}$.

Consider that the NOMA decoding principle requires the symbol with more allocated power to be decoded first. In order to successfully and simultaneously decode $x_{1}$ and $x_{2}$ at the relay $i$, the SIC technique is utilized. Obtaining the transmitted signals in this manner, the reception SNRs for $x_{1}$ and $x_{2}$ at the relay $i$ can be respectively expressed as

$$
\gamma_{R_{i}}^{\left(x_{1}\right)}=\frac{\left|h_{S R_{i}}\right|^{2} a_{1} \rho}{\left|h_{S R_{i}}\right|^{2} a_{2} \rho+1}, \gamma_{R_{i}}^{\left(x_{2}\right)}=\left|h_{S R_{i}}\right|^{2} a_{2} \rho
$$

where $\rho=\frac{P_{t}}{\sigma_{R_{i}}^{2}}$ is the transmission SNR.

\subsection{Proposed CTRS-NOMA superposition case}

At the second time slot of the proposed CTRS-NOMA superposition case, both of two relay nodes forward the superposition-coded signal $x_{R}$ to the destination

$$
x_{R}=\sqrt{b_{1}} x_{1}+\sqrt{b_{2}} x_{2},
$$

where $b_{i}$, with $b_{1}+b_{2}=0.5$ and $b_{1}>b_{2}$, is the new power allocation coefficient, where the similar PA assumption of $a_{i}$ is considered. By utilizing a MRC reception at the destination, the corresponding received SNR for $x_{1}$ and $x_{2}$ are given as

$$
\gamma_{D}^{\left(x_{1}\right)}=\frac{b_{1}\left|h_{R_{1} D}\right|^{2} \rho}{b_{2}\left|h_{R_{1} D}\right|^{2} \rho+1}+\frac{b_{1}\left|h_{R_{2} D}\right|^{2} \rho}{b_{2}\left|h_{R_{2} D}\right|^{2} \rho+1},
$$

and

$$
\gamma_{D}^{\left(x_{2}\right)}=b_{2}\left|h_{R_{1} D}\right|^{2} \rho+b_{2}\left|h_{R_{2} D}\right|^{2} \rho .
$$




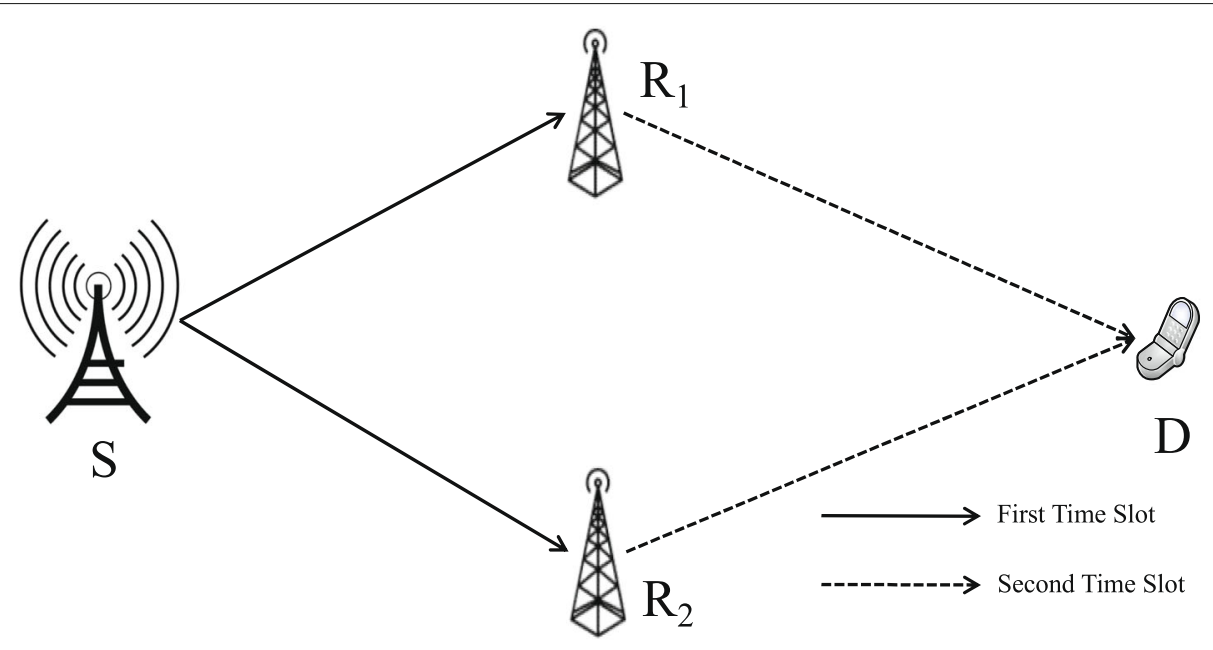

Fig. 1 The proposed cooperative two relaying system with NOMA

Therefore, the achievable SR for the proposed CRSNOMA can be obtained from

$$
\begin{aligned}
C_{\text {sum }}= & \frac{1}{2} \log _{2}\left(1+\min \left\{\gamma_{R_{1}}^{\left(x_{1}\right)}, \gamma_{R_{2}}^{\left(x_{1}\right)}, \gamma_{D}^{\left(x_{1}\right)}\right\}\right) \\
& +\frac{1}{2} \log _{2}\left(1+\min \left\{\gamma_{R_{1}}^{\left(x_{2}\right)}, \gamma_{R_{2}}^{\left(x_{2}\right)}, \gamma_{D}^{\left(x_{2}\right)}\right\}\right) .
\end{aligned}
$$

\subsection{Proposed CTRS-NOMA single case}

In this case, we further investigate a relay transmission method considered in [39] for our proposed CTRSNOMA system as a benchmark. After a signal reception from the source, two relay nodes simultaneously DF the signals to the destination node during the second time slot. In this scenario, we assume that the relay node $i$ forwards the signal $x_{i}$ to the destination. The received signal at destination can be expressed as

$$
y_{D}=\sqrt{c_{i}} h_{R_{1} D} x_{i}+\sqrt{c_{\bar{i}}} h_{R_{2} D} x_{\bar{i}}+n_{D}
$$

where $n_{D} \sim \mathcal{C N}\left(0, \sigma^{2}\right)$ denotes AWGN with zero mean and variance $\sigma_{R_{D}}^{2}$ and $c_{i}$ is the new PA factor with $c_{1}+c_{2}=$ 1 and $c_{1}>c_{2}$.

Case 1: If $x_{1}$ is allocated with PA factor $c_{1}$, the corresponding received SNR for $x_{1}$ and $x_{2}$ are given as

$$
\gamma_{I}^{\left(x_{1}\right)}=\frac{c_{1}\left|h_{R_{1} D}\right|^{2} \rho}{c_{2}\left|h_{R_{2} D}\right|^{2} \rho+1}, \gamma_{I}^{\left(x_{2}\right)}=c_{2}\left|h_{R_{2} D}\right|^{2} \rho,
$$

with the achievable SR for the proposed CTRS-NOMA single case I can be obtained from

$$
\begin{aligned}
C^{(I)}= & \frac{1}{2} \log _{2}\left(1+\min \left\{\gamma_{R_{1}}^{\left(x_{1}\right)}, \gamma_{R_{2}}^{\left(x_{1}\right)}, \gamma_{I}^{\left(x_{1}\right)}\right\}\right) \\
& +\frac{1}{2} \log _{2}\left(1+\min \left\{\gamma_{R_{1}}^{\left(x_{2}\right)}, \gamma_{R_{2}}^{\left(x_{2}\right)}, \gamma_{I}^{\left(x_{2}\right)}\right\}\right) .
\end{aligned}
$$

Case 2: Without loss of the generality, if $x_{2}$ is allocated with PA factor $c_{1}$, the corresponding received SNR for $x_{1}$ and $x_{2}$ are given as

$$
\gamma_{I I}^{\left(x_{1}\right)}=c_{2}\left|h_{R_{1} D}\right|^{2} \rho, \gamma_{I I}^{\left(x_{2}\right)}=\frac{c_{1}\left|h_{R_{2} D}\right|^{2} \rho}{c_{2}\left|h_{R_{1} D}\right|^{2} \rho+1},
$$

with the achievable SR for the proposed CTRS-NOMA single case II as

$$
\begin{aligned}
C^{(I I)}= & \frac{1}{2} \log _{2}\left(1+\min \left\{\gamma_{R_{1}}^{\left(x_{1}\right)}, \gamma_{R_{2}}^{\left(x_{1}\right)}, \gamma_{I I}^{\left(x_{1}\right)}\right\}\right) \\
& +\frac{1}{2} \log _{2}\left(1+\min \left\{\gamma_{R_{1}}^{\left(x_{2}\right)}, \gamma_{R_{2}}^{\left(x_{2}\right)}, \gamma_{I I}^{\left(x_{2}\right)}\right\}\right) .
\end{aligned}
$$

\section{Achievable ergodic SR analysis}

In this section, the achievable ergodic SR of our proposed CTRS-NOMA system is analyzed in detail; the closedform expressions are obtained for the achievable ergodic SRs assuming that each independent channel undergoes Rayleigh fading.

\subsection{Achievable SR for the proposed CTRS-NOMA single case I}

Further denoting $\left|h_{S R_{i}}\right|^{2}=\beta_{S R_{i}}$ and $\left|h_{R_{i} D}\right|^{2}=\beta_{R_{i} D}$, we have

$$
\begin{aligned}
\min \left\{\gamma_{R_{1}}^{\left(x_{1}\right)}, \gamma_{R_{2}}^{\left(x_{1}\right)}, \gamma_{I}^{\left(x_{1}\right)}\right\}= & \min \left\{\frac{\beta_{S R_{1}} a_{1} \rho}{\beta_{S R_{1}} a_{2} \rho+1}, \frac{\beta_{S R_{2}} a_{1} \rho}{\beta_{S R_{2}} a_{2} \rho+1},\right. \\
& \left.\frac{\beta_{R_{1} D} c_{1} \rho}{\beta_{R_{2} D} c_{2} \rho+1}\right\},
\end{aligned}
$$

Letting $X=\min \left\{\gamma_{R_{1}}^{\left(x_{1}\right)}, \gamma_{R_{2}}^{\left(x_{1}\right)}, \gamma_{I}^{\left(x_{1}\right)}\right\}$, the complementary cumulative distribution function (CCDF) of $X$ can be obtained as

$$
\begin{aligned}
\bar{F}_{X}(x)= & \operatorname{Pr}\left\{\frac{\beta_{S R_{1}} a_{1} \rho}{\beta_{S R_{1}} a_{2} \rho+1}>x, \frac{\beta_{S R_{2}} a_{1} \rho}{\beta_{S R_{2}} a_{2} \rho+1}\right. \\
& \left.>x, \frac{\beta_{R_{1} D} c_{1} \rho}{\beta_{R_{2} D c_{2} \rho+1} \rho}>x\right\} .
\end{aligned}
$$


Noting that the CCDF of $\beta_{\delta}=e^{-\frac{x}{\alpha_{\delta}}}$, for $\delta \in\left\{S R_{i}, R_{i} D\right\}$, when $x<\frac{a_{1}}{a_{2}}$, (13) can be equivalently represented as

$$
\begin{aligned}
& \bar{F}_{X}(x)=\left[E_{\beta_{R_{2} D}}\left[\operatorname{Pr}\left\{\beta_{R_{1} D}>\left(\frac{c_{2} \beta_{R_{2} D}}{c_{1}}+\frac{1}{c_{1} \rho}\right) x\right\}\right]\right] \bar{F}_{S R_{1}}\left(\frac{x}{a_{1} \rho-a_{2} \rho x}\right) \\
& \times \bar{F}_{S R_{2}}\left(\frac{x}{a_{1} \rho-a_{2} \rho x}\right) \\
& =\frac{1}{\alpha_{R_{2} D}} e^{-\frac{x}{a_{1} \rho-a_{2} \rho x}\left(\frac{1}{\alpha_{S R_{1}}}+\frac{1}{\alpha_{S R_{2}}}\right)-\frac{x}{c_{1} \rho \alpha_{1} D}} \int_{0}^{\infty} e^{-u\left(\frac{c_{2} x}{c_{1} \alpha_{1} D}+\frac{1}{\alpha_{R_{2} D} D}\right)} d u \\
& =\frac{c_{1} \alpha_{R_{1} D}}{c_{2} \alpha_{R_{2} D} x+c_{1} \alpha_{R_{1} D}} e^{-\frac{x}{a_{1} \rho-a_{2} \rho x}\left(\frac{1}{\alpha_{S R_{1}}}+\frac{1}{\alpha_{S R_{2}}}\right)-\frac{x}{c_{1} \rho \alpha_{R_{1}} D}}
\end{aligned}
$$

where $E[\cdot]$ stands for the statistical expectation. For the case $x>\frac{a_{1}}{a_{2}}, \bar{F}_{X}(x)=0$ always holds due to

$$
\frac{\beta_{S R_{1}} a_{1} \rho}{\beta_{S R_{1}} a_{2} \rho+1}<\frac{a_{1}}{a_{2}} .
$$

Since the derivatives of (14) with respect to $\frac{1}{1+x}$ is quite involved, we try to find an approximation result by considering the high transmission SNR, i.e., $\rho \gg 0$, as follows

$$
\bar{F}_{X}(x) \sim \frac{c_{1} \alpha_{R_{1} D}}{c_{2} \alpha_{R_{2} D} x+c_{1} \alpha_{R_{1} D}} e^{-\frac{x}{c_{1} \rho \alpha_{R_{1}} D}} \text {, for } x<\frac{a_{1}}{a_{2}} .
$$

With (16) and using the equality

$$
\int_{0}^{\infty} \log _{2}(1+x) f_{X}(x) d x=\frac{1}{2 \ln 2} \int_{0}^{\infty} \frac{1-F(x)}{1+x} d x,(17)
$$

for the high SNR case, the achievable ergodic rate for $x_{1}$ can be obtained as follows

$$
\begin{aligned}
& C_{I}^{\left(x_{1}\right)} \\
& =\int_{0}^{\frac{a_{1}}{a_{2}}} \frac{1}{2} \log _{2}(1+x) d F_{X}(x)+\frac{1}{2} \log _{2}\left(1+\frac{a_{1}}{a_{2}}\right)\left(1-F_{X}\left(\frac{a_{1}}{a_{2}}\right)\right) \\
& =\frac{1}{2} \log _{2}\left(1+\frac{a_{1}}{a_{2}}\right)-\frac{1}{2 \ln 2} \int_{0}^{\frac{a_{1}}{a_{2}}} \frac{1}{1+x}\left(1-\frac{c_{1} \alpha_{R_{1} D}}{c_{2} \alpha_{R_{2}} D^{x}+c_{1} \alpha_{R_{1}} D} e^{-\frac{x}{c_{1} \rho R_{1} D}}\right) d x \\
& =\frac{1}{2 \ln 2} \int_{0}^{\frac{a_{1}}{a_{2}}} \frac{c_{1} \alpha_{R_{1} D}}{c_{2} \alpha_{R_{2}} D^{x}+c_{1} \alpha_{R_{1} D}} \frac{e^{-\frac{x}{c_{1} \rho \alpha_{1} D}}}{1+x} d x \\
& =\frac{1}{2 \ln 2} \frac{c_{1} \alpha_{R_{1} D}}{c_{2} \alpha_{R_{2} D}-c_{1} \alpha_{R_{1} D}}\left(e^{\frac{1}{c_{1} \rho \alpha_{1} D}}\left[\operatorname{Ei}\left(-\frac{1}{c_{1} \rho \alpha_{R_{1}} D}\right)-\operatorname{Ei}\left(-\frac{1}{a_{2} c_{1} \rho \alpha_{R_{1}} D}\right)\right]\right. \\
& \left.-e^{\frac{1}{c_{2} \rho \alpha_{R_{2} D}}}\left[\operatorname{Ei}\left(-\frac{1}{c_{2} \rho \alpha_{R_{2} D}}\right)-\operatorname{Ei}\left(-\frac{1}{c_{2} \rho \alpha_{R_{2} D}}-\frac{a_{1}}{a_{2} c_{1} \rho \alpha_{R_{1} D}}\right)\right]\right),
\end{aligned}
$$

where the second term in (18) is simplified by using

$$
\int_{0}^{u} \frac{e^{-\mu x} d x}{x+\beta}=e^{\mu \beta}[\operatorname{Ei}(-\mu u-\mu \beta)-\operatorname{Ei}(-\mu \beta)]
$$

[42, Eq. (3.352.1)], and $\mathrm{Ei}(\cdot)$ denotes the exponential integral function.
Correspondingly, letting $Y=\min \left\{\gamma_{R_{1}}^{\left(x_{2}\right)}, \gamma_{R_{2}}^{\left(x_{2}\right)}, \gamma_{I}^{\left(x_{2}\right)}\right\}$, CCDF of $Y$ can be obtained as

$$
\begin{aligned}
\bar{F}_{Y}(y) & =\left\{\beta_{S R_{1}} a_{2} \rho>y, \beta_{S R_{2}} a_{2} \rho>y, c_{2} \beta_{R_{2} D} \rho>y\right\} \\
& =\bar{F}_{S R_{1}}\left(\frac{y}{a_{2} \rho}\right) \bar{F}_{S R_{2}}\left(\frac{y}{a_{2} \rho}\right) \bar{F}_{R_{2} D}\left(\frac{y}{c_{2} \rho}\right) \\
& =e^{-\frac{y}{c_{2} \rho R_{2} D}-\frac{y}{a_{2} \rho \alpha R_{1}}-\frac{y}{a_{2} \rho \alpha R_{2}}} .
\end{aligned}
$$

By taking derivative of (20), the PDF of $Y$ can be obtained as

$$
\begin{aligned}
f_{Y}(y)= & \left(\frac{1}{c_{2} \rho \alpha_{R_{2} D}}+\frac{1}{a_{2} \rho \alpha_{S R_{1}}}+\frac{1}{a_{2} \rho \alpha_{S R_{2}}}\right) \\
& e^{-\frac{y}{c_{2} \rho \alpha R_{2} D}-\frac{y}{a_{2} \rho \alpha S R_{1}}-\frac{y}{a_{2} \rho \alpha S R_{2}}} .
\end{aligned}
$$

From (20) and (21), the achievable ergodic rate for $x_{2}$ can be calculated as

$$
\begin{aligned}
& C_{I}^{\left(x_{2}\right)}=\frac{1}{2 \ln 2} \int_{0}^{\infty} \frac{e^{-\frac{y}{c_{2} \rho \alpha R_{2} D}-\frac{y}{a_{2} \rho \alpha} S R_{1}}-\frac{y}{a_{2} \rho \alpha S R_{2}}}{1+x} d y \\
& =\frac{e^{\frac{1}{c_{2} \rho \alpha_{R_{2}} D}+\frac{1}{a_{2} \rho \alpha S R_{1}}+\frac{1}{a_{2} \rho \alpha S R_{2}}}}{2 \ln 2} \operatorname{Ei}\left(-\frac{1}{c_{2} \rho \alpha_{R_{2} D}}\right. \\
& \left.-\frac{1}{a_{2} \rho \alpha_{S R_{1}}}-\frac{1}{a_{2} \rho \alpha_{S R_{2}}}\right) \text {. }
\end{aligned}
$$

where the integral result

$$
\int_{0}^{\infty} \frac{e^{-\mu x} d x}{x+\beta}=e^{\mu \beta} \operatorname{Ei}(-\mu \beta)
$$

[42, Eq. (3.352.4)] is used. Combing (18) and (22), the achievable SR can be finally expressed as

$C^{(I)}$

$$
\begin{array}{r}
=\frac{1}{2 \ln 2} \frac{c_{1} \alpha_{R_{1} D}}{c_{2} \alpha_{R_{2} D}-c_{1} \alpha_{R_{1} D}}\left(e ^ { \frac { 1 } { c _ { 1 } \rho \alpha _ { R _ { 1 } } D } } \left[\operatorname{Ei}\left(-\frac{1}{c_{1} \rho \alpha_{R_{1} D}}\right)\right.\right. \\
-\operatorname{Ei}\left(-\frac{1}{a_{1} c_{1} \rho \alpha_{R_{1} D}}\right) \\
-e^{\frac{1}{c_{2} \rho \alpha_{R_{2}} D}}\left[\operatorname{Ei}\left(-\frac{1}{c_{2} \rho \alpha_{R_{2} D}}\right)-\operatorname{Ei}\left(-\frac{1}{c_{2} \rho \alpha_{R_{2} D}}\right.\right. \\
\left.\left.\left.-\frac{a_{2}}{a_{1} c_{1} \rho \alpha_{R_{1} D}}\right)\right]\right) \\
+\frac{1}{2 \ln 2} e^{\frac{1}{b_{2} \rho \alpha_{R_{2} D}}+\frac{1}{a_{2} \rho \alpha S R_{1}}+\frac{1}{a_{2} \rho \alpha_{S R_{2}}}} \operatorname{Ei}\left(-\frac{1}{b_{2} \rho \alpha_{R_{2} D}}-\frac{1}{a_{2} \rho \alpha_{S R_{1}}}\right. \\
\left.-\frac{1}{a_{2} \rho \alpha_{S R_{2}}}\right) .
\end{array}
$$




\subsection{Achievable SR for the proposed CTRS-NOMA single} case 2

Denoting $\mathcal{J}=\min \left\{\gamma_{R_{1}}^{\left(x_{1}\right)}, \gamma_{R_{2}}^{\left(x_{1}\right)}, \gamma_{I I}^{\left(x_{1}\right)}\right\}$, the CCDF of $\mathcal{J}$ can be obtained as

$$
\begin{aligned}
\bar{F}_{\mathcal{J}}(j)=\operatorname{Pr} & \left\{\frac{\beta_{S R_{1}} a_{1} \rho}{\beta_{S R_{1}} a_{2} \rho+1}>\mathcal{J}, \frac{\beta_{S R_{2}} a_{1} \rho}{\beta_{S R_{2}} a_{2} \rho+1}\right. \\
>\mathcal{J}, c_{2} \beta_{R_{1} D} \rho>\mathcal{J} & \left.\frac{\beta_{S R_{1}} a_{1} \rho}{\beta_{S R_{1}} a_{2} \rho+1}\right\} .
\end{aligned}
$$

Considering a high SNR case, the closed-form expression of $\mathcal{J}$ is given as

$$
\bar{F}_{\mathcal{J}}(j) \sim e^{-\frac{j}{c_{2} \rho R_{R_{1} D}}}, \text { for } j<\frac{a_{1}}{a_{2}}
$$

with the corresponding ergodic rate as

$$
\begin{aligned}
C_{I I}^{\left(x_{1}\right)}= & \frac{1}{2 \ln 2} \int_{0}^{\frac{a_{1}}{a_{2}}} \frac{e^{-\frac{j}{c \alpha_{2} \rho R_{1} D}}}{1+j} d j \\
= & \frac{1}{2 \ln 2} e^{\frac{1}{c_{1} \rho \alpha_{R_{1} D}}}\left[\operatorname{Ei}\left(-\frac{1}{a_{2} c_{2} \rho \alpha_{R_{1} D}}\right)\right. \\
& \left.-\operatorname{Ei}\left(-\frac{1}{c_{2} \rho \alpha_{R_{1} D}}\right)\right],
\end{aligned}
$$

where where $\int_{0}^{u} \frac{e^{-\mu x} d x}{x+\beta}=e^{\mu \beta}[\operatorname{Ei}(-\mu u-\mu \beta)-\operatorname{Ei}(-\mu \beta)]$ [42, Eq. (3.352.1)] is used.

On the other hand, for $\mathcal{T}=\min \left\{\gamma_{R_{1}}^{\left(x_{2}\right)}, \gamma_{R_{2}}^{\left(x_{2}\right)}, \gamma_{I I}^{\left(x_{2}\right)}\right\}$, the CCDF of $\mathcal{T}$ can be obtained as follows

$$
\begin{aligned}
\bar{F}_{\mathcal{T}}(t)= & \left\{\beta_{S R_{1}} a_{2} \rho>\mathcal{T}, \beta_{S R_{2}} a_{2} \rho>\mathcal{T}, \frac{c_{1} \beta_{R_{2} D} \rho}{c_{2} \beta_{R_{1}} D+1}>\mathcal{T}\right\} \\
= & \bar{F}_{S R_{1}}\left(\frac{t}{a_{2} \rho}\right) \bar{F}_{S R_{2}}\left(\frac{t}{a_{2} \rho}\right)\left[E _ { \beta _ { R _ { 1 } } D } \left[\operatorname { P r } \left\{\beta_{R_{2} D}>\left(\frac{c_{2} \beta_{R_{1} D}}{c_{1}}\right.\right.\right.\right. \\
& \left.\left.\left.\left.+\frac{1}{c_{1} \rho}\right) t\right\}\right]\right] \\
= & \frac{1}{\alpha_{R_{1} D} D} e^{-\frac{t}{a_{2} \rho}\left(\frac{1}{\alpha_{S R_{1}}}+\frac{1}{\alpha_{S R_{2}}}\right)-\frac{t}{c_{1} \rho \alpha_{R_{2} D}}} \int_{0}^{\infty} e^{-u\left(\frac{c_{2} t}{c_{1} \alpha_{R_{2} D} D}+\frac{1}{\alpha_{R_{1} D} D}\right)} d u \\
= & \frac{c_{1} \alpha_{R_{2} D}}{c_{2} \alpha_{R_{1} D} t+c_{1} \alpha_{R_{2} D}} e^{-\frac{t}{a_{2} \rho}\left(\frac{1}{\alpha_{S R_{1}}}+\frac{1}{\alpha_{S R_{2}}}\right)-\frac{t}{c_{1} \rho \alpha_{R_{2} D}}},
\end{aligned}
$$

and the corresponding closed-form expression for the ergodic rate of $x_{2}$ can be written as follows

$$
\begin{aligned}
C_{I I}^{\left(x_{2}\right)} & =\frac{1}{2 \ln 2} \int_{0}^{\infty} \frac{c_{1} \alpha_{R_{2} D}}{c_{2} \alpha_{R_{1} D} t+c_{1} \alpha_{R_{2} D}} \\
& \frac{e^{-\frac{t}{a_{2} \rho}\left(\frac{1}{\alpha_{S R_{1}}}+\frac{1}{\alpha_{S R_{2}}}\right)-\frac{t}{c_{1} \rho \alpha_{R_{2} D} D}}}{1+t} d t \\
& =\frac{1}{2 \ln 2} \frac{c_{1} \alpha_{R_{2} D}}{c_{1} \alpha_{R_{2} D}-c_{2} \alpha_{R_{1} D}}\left[e^{\mathcal{K}_{1}} \operatorname{Ei}\left(-\mathcal{K}_{1}\right)-e^{\frac{c_{1} \alpha_{R_{2} D} \mathcal{K}_{1}}{c_{2} \alpha_{R_{1}} D}}\right. \\
& \left.\operatorname{Ei}\left(-\frac{c_{1} \alpha_{R_{2} D} \mathcal{K}_{1}}{c_{2} \alpha_{R_{1} D}}\right)\right],
\end{aligned}
$$

where $\mathcal{K}_{1}=\frac{1}{a_{2} \rho}\left(\frac{1}{\alpha_{S R_{1}}}+\frac{1}{\alpha_{S R_{2}}}\right)+\frac{1}{c_{1} \rho \alpha_{R_{2} D}}$. Synthesizing (27) and (29), the achievable SR can be expressed as

$$
\begin{aligned}
C^{(I I)}= & \frac{1}{2 \ln 2} e^{\frac{1}{c_{1} \rho \alpha_{R_{1} D} D}}\left[\operatorname{Ei}\left(-\frac{1}{a_{2} c_{2} \rho \alpha_{R_{1} D}}\right)\right. \\
& \left.-\operatorname{Ei}\left(-\frac{1}{c_{2} \rho \alpha_{R_{1} D} D}\right)\right] \\
& +\frac{1}{2 \ln 2} \frac{c_{1} \alpha_{R_{2} D}}{c_{1} \alpha_{R_{2} D}-c_{2} \alpha_{R_{1} D}}\left[e^{\mathcal{K}_{1}} \operatorname{Ei}\left(-\mathcal{K}_{1}\right)-e^{\frac{c_{1} \alpha_{R_{2} D} \mathcal{K}_{1}}{c_{2} \alpha_{R_{1}} D}}\right. \\
& \left.\operatorname{Ei}\left(-\frac{c_{1} \alpha_{R_{2} D} \mathcal{K}_{1}}{c_{2} \alpha_{R_{1} D}}\right)\right] .
\end{aligned}
$$

\subsection{Achievable SR for the proposed CTRS-NOMA superposition case}

For our proposed CRS-NOMA scheme, the corresponding SNRs for $x_{1}$ are given as

$$
\begin{aligned}
& \min \left\{\gamma_{R_{1}}^{\left(x_{1}\right)}, \gamma_{R_{2}}^{\left(x_{1}\right)}, \gamma_{D}^{\left(x_{1}\right)}\right\} \\
&= \min \left\{\frac{\beta_{S R_{1}} a_{1} \rho}{\beta_{S R_{1}} a_{2} \rho+1}, \frac{\beta_{S R_{2}} a_{1} \rho}{\beta_{S R_{2}} a_{2} \rho+1}, \frac{b_{1}\left|h_{R_{1} D}\right|^{2} \rho}{b_{2}\left|h_{R_{1} D}\right|^{2} \rho+1}\right. \\
&\left.+\frac{b_{1}\left|h_{R_{2} D}\right|^{2} \rho}{b_{2}\left|h_{R_{2} D}\right|^{2} \rho+1}\right\} .
\end{aligned}
$$

Assuming $\mathcal{V}=\min \left\{\gamma_{R_{1}}^{\left(x_{1}\right)}, \gamma_{R_{2}}^{\left(x_{1}\right)}, \gamma_{D}^{\left(x_{1}\right)}\right\}$, the CCDF of $\mathcal{V}$ can be obtained from

$$
\begin{aligned}
& \bar{F}_{\mathcal{V}}(v) \\
& =\min \left\{\frac{\beta_{S R_{1}} a_{1} \rho}{\beta_{S R_{1}} a_{2} \rho+1}>v, \frac{\beta_{S R_{2}} a_{1} \rho}{\beta_{S R_{2}} a_{2} \rho+1}\right. \\
& \left.>v, \frac{b_{1}\left|h_{R_{1} D}\right|^{2} \rho}{b_{2}\left|h_{R_{1} D}\right|^{2} \rho+1}+\frac{b_{1}\left|h_{R_{2} D}\right|^{2} \rho}{b_{2}\left|h_{R_{2} D}\right|^{2} \rho+1}>v\right\} .
\end{aligned}
$$




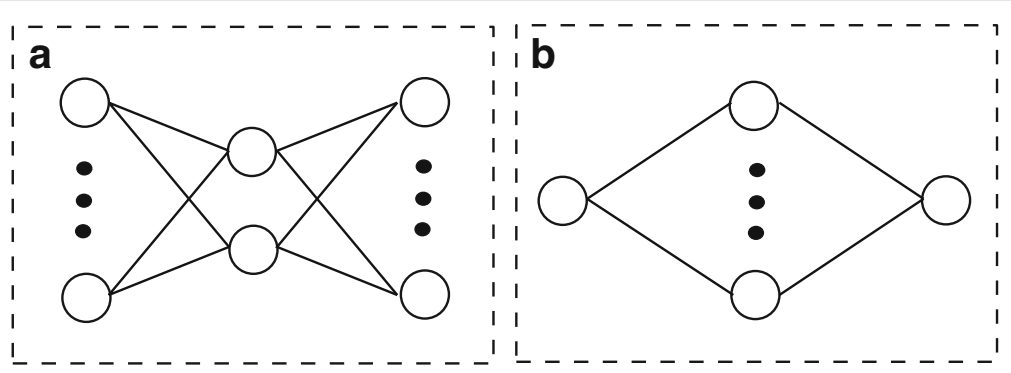

Fig. 2 The extended system model. a The $K$ pairs of source nodes scenario. $\mathbf{b}$ The $T$ relay nodes scenario

It is clear that the derivative of (31) is quite involved. We turn to an approximation of it for the higher SNRs. With the approximations

$$
\begin{aligned}
& \frac{b_{1}\left|h_{R_{1} D}\right|^{2} \rho}{b_{2}\left|h_{R_{1} D}\right|^{2} \rho+1}+\frac{b_{1}\left|h_{R_{2} D}\right|^{2} \rho}{b_{2}\left|h_{R_{2} D}\right|^{2} \rho+1} \sim \frac{2 b 1}{b_{2}}, \\
& \frac{\beta_{S R_{1}} a_{1} \rho}{\beta_{S R_{1}} a_{2} \rho+1} \sim \frac{a_{1}}{a_{2}}, \text { and } \frac{\beta_{S R_{2}} a_{1} \rho}{\beta_{S R_{2}} a_{2} \rho+1} \sim \frac{a_{1}}{a_{2}}
\end{aligned}
$$

for $\rho \gg 1$, we have

$$
C_{D}^{\left(x_{1}\right)} \sim \frac{1}{2} \log _{2}\left(1+\min \left\{\frac{a_{1}}{a_{2}}, \frac{2 b_{1}}{b_{2}}\right\}\right) .
$$

Otherwise $C_{D}^{\left(x_{1}\right)}=0, \frac{\beta_{S R_{1}} a_{1} \rho}{\beta_{S R_{1}} a_{2} \rho+1}<\frac{a_{1}}{a_{2}}, \frac{\beta_{S R_{2}} a_{1} \rho}{\beta_{S R_{2}} a_{2} \rho+1}<\frac{a_{1}}{a_{2}}$, and $\frac{b_{1} \beta_{R_{1} D \rho}}{b_{2} \beta_{R_{1} D \rho+1} \rho+1}+\frac{b_{1} \beta_{R_{2} D \rho}}{b_{2} \beta_{R_{2} D \rho+1}}<\frac{2 b_{1}}{b_{2}}$ always hold.

On the other hand, for $x_{2}$, letting

$$
\mathcal{G}=\min \left\{\gamma_{R_{1}}^{\left(x_{2}\right)}, \gamma_{R_{2}}^{\left(x_{2}\right)}, \gamma_{D}^{\left(x_{2}\right)}\right\}
$$

the $\mathrm{CCDF}$ of $\mathcal{G}$ can be obtained from

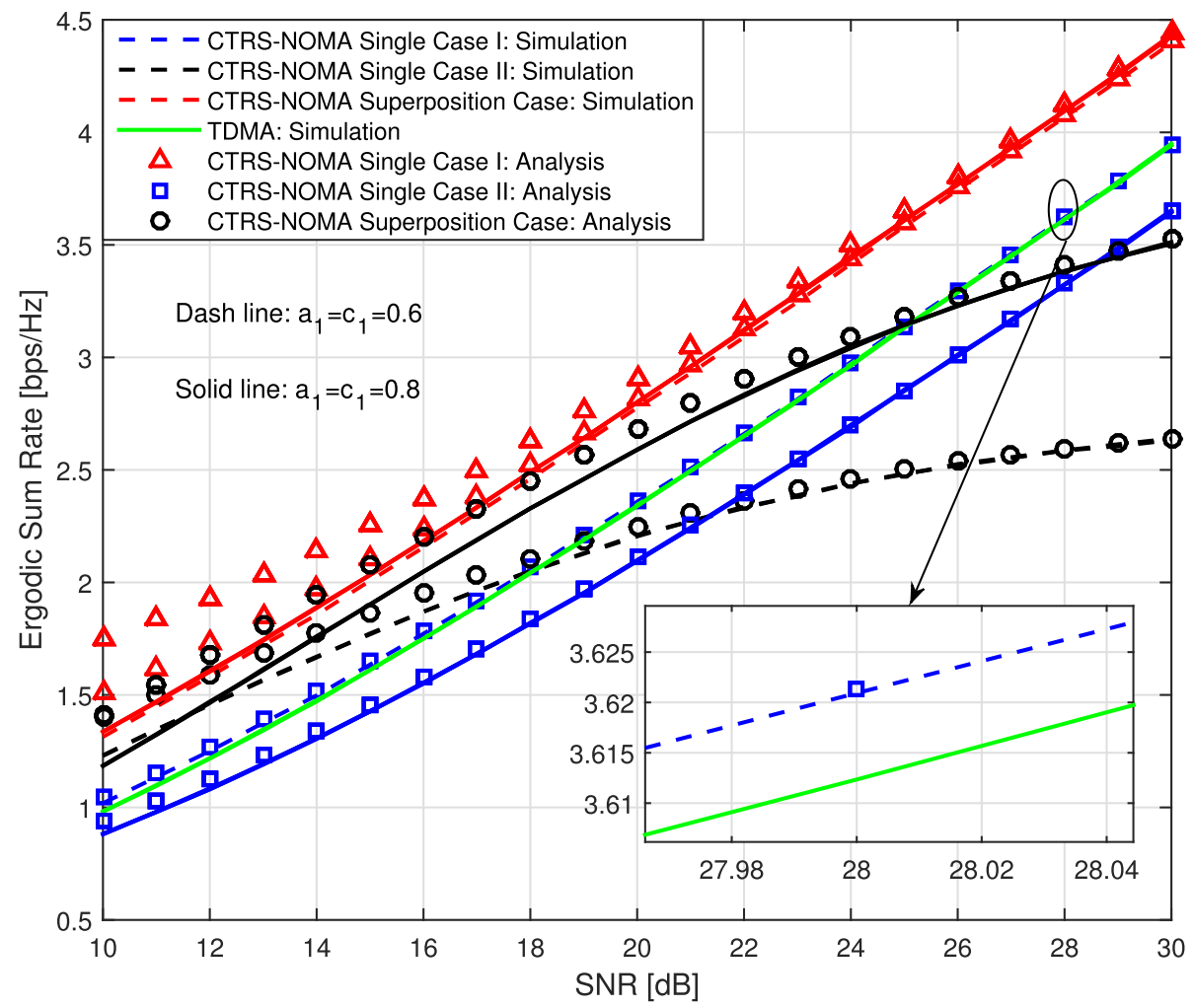

Fig. 3 The ergodic SRs achieved by our proposed CTRS-NOMA single case I, CTRS-NOMA single case II, CTRS-NOMA superposition case, and the TDMA scheme with fixed $\alpha_{S R_{1}}=5, \alpha_{S R_{2}}=1, \alpha_{R_{1} D}=2, \alpha_{R_{2} D}=20, b_{1}=0.4$, and $a_{1}=c_{1}=\{0.6,0.8\}$ versus the transmission SNR 


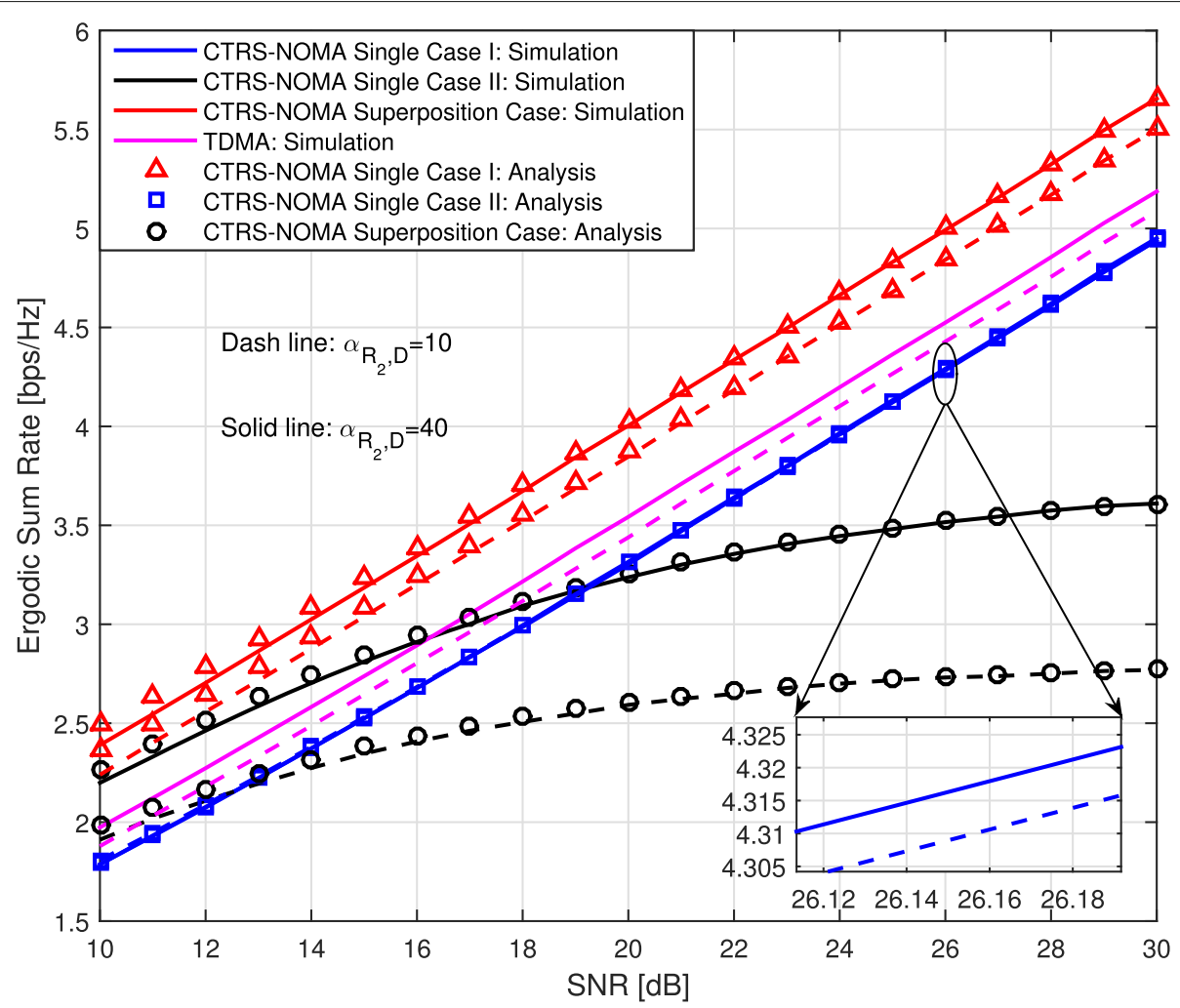

Fig. 4 The ergodic SRs achieved by our proposed CTRS-NOMA single case I, CTRS-NOMA single case II, CTRS-NOMA superposition case, and the TDMA scheme with fixed $a_{1}=c_{1}=0.7, b_{1}=0.3, \alpha_{S R_{1}}=\alpha_{S R_{2}}=10, \alpha_{R_{1} D}=2$, and $\alpha_{R_{2} D}=\{10,40\}$ versus the transmission SNR

$$
\begin{aligned}
& \bar{F}_{\mathcal{G}}(g)=\operatorname{Pr}\left\{\beta_{S R_{1}} a_{2} \rho>g, \beta_{S R_{2}} a_{2} \rho>g, \beta_{R_{1} D} b_{2} \rho+\beta_{R_{2} D} b_{2} \rho>g\right\} \\
& =\bar{F}_{\beta_{S R_{1}}}\left(\frac{g}{a_{2} \rho}\right) \bar{F}_{\beta_{S R_{2}}}\left(\frac{g}{a_{2} \rho}\right)\left[E _ { \beta _ { R _ { 2 } D } } \left[\operatorname { P r } \left\{\beta_{R_{1} D}>\frac{g}{b_{2} \rho}\right.\right.\right. \\
& \left.\left.\left.-\beta_{R_{2} D}\right\}\right]\right] \\
& =\frac{1}{\alpha_{R_{2} D}} e^{-\frac{g}{b_{2} \rho \alpha R_{1} D}-\frac{g}{a_{2} \rho \alpha S R_{1}}-\frac{g}{a_{2} \rho \alpha} S R_{2}} \int_{0}^{\frac{g}{b_{2} \rho}} e^{\frac{u}{\alpha_{1} D}-\frac{u}{\alpha_{R_{2} D}}} d u
\end{aligned}
$$

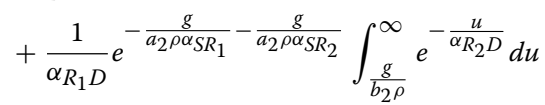

$$
\begin{aligned}
& =\frac{\alpha_{R_{1} D}}{\alpha_{R_{1} D}-\alpha_{R_{2} D}} e^{-\frac{g}{b_{2} \rho \alpha_{R_{1} D}}-\frac{g}{a_{2} \rho \alpha S R_{1}}-\frac{g}{a_{2} \rho \alpha S R_{2}}} \\
& +\frac{\alpha_{R_{2} D}}{\alpha_{R_{1} D}-\alpha_{R_{2} D}} e^{-\frac{g}{a_{2} \rho \alpha} S R_{1}}-\frac{g}{a_{2} \rho \alpha} S R_{2}-\frac{g}{b_{2} \rho \alpha R_{2} D} .
\end{aligned}
$$

Therefore, letting $\mathcal{D}_{1}=-\frac{1}{b_{2} \rho \alpha_{R_{1} D}}-\frac{1}{a_{2} \rho \alpha_{S R_{1}}}-\frac{1}{a_{2} \rho \alpha_{S R_{2}}}$ and $\mathcal{D}_{2}=-\frac{1}{a_{2} \rho \alpha_{S R_{1}}}-\frac{1}{a_{2} \rho \alpha_{S R_{2}}}-\frac{1}{b_{2} \rho \alpha_{R_{2} D}}$, substituting (35) back into (17), the closed form of ergodic rate for $x_{2}$ is given as

$$
\begin{aligned}
C_{D}^{\left(x_{2}\right)} & =\frac{1}{2 \ln 2} \int_{0}^{\infty} \frac{\frac{\alpha_{R_{1} D}}{\alpha_{R_{1} D}-\alpha_{R_{2} D}} e^{\mathcal{D}_{1} g}+\frac{\alpha_{R_{2} D}}{\alpha_{R_{1} D}-\alpha_{R_{2} D}} e^{\mathcal{D}_{2} g}}{1+g} d g \\
& =\frac{\alpha_{R_{1} D} e^{-\mathcal{D}_{1}} \operatorname{Ei~}\left(\mathcal{D}_{1}\right)}{2 \ln 2\left(\alpha_{R_{1} D}-\alpha_{R_{2} D}\right)}-\frac{\alpha_{R_{2} D} e^{-\mathcal{D}_{2}} \operatorname{Ei}\left(\mathcal{D}_{2}\right)}{2 \ln 2\left(\alpha_{R_{1} D}-\alpha_{R_{2} D}\right)},
\end{aligned}
$$

where $\int_{0}^{\infty} \frac{e^{-\mu x} d x}{x+\beta}=e^{\mu \beta} \operatorname{Ei}(-\mu \beta)[42$, Eq. (3.352.4)] is used in the second term. Finally, the closed-form expression of SR for the conventional NOMA can be denoted as

$$
\begin{aligned}
C_{D}^{\text {sum }}= & \frac{\alpha_{R_{1} D} e^{-\mathcal{D}_{1}} \operatorname{Ei}\left(\mathcal{D}_{1}\right)}{2 \ln 2\left(\alpha_{R_{1} D}-\alpha_{R_{2} D}\right)}-\frac{\alpha_{R_{2} D} e^{-\mathcal{D}_{2}} \operatorname{Ei}\left(\mathcal{D}_{2}\right)}{2 \ln 2\left(\alpha_{R_{1} D}-\alpha_{R_{2} D}\right)} \\
& +\frac{1}{2} \log _{2}\left(1+\min \left\{\frac{a_{1}}{a_{2}}, \frac{2 b_{1}}{b_{2}}\right\}\right) .
\end{aligned}
$$

Discussion: Figure 2 displays two extended system models. One is the multipair scenario with two relay nodes and $K$ pairs of source nodes while the other is $Z$ relay nodes scenario with two source nodes, where $Z$ is an even integer with $Z \geq 2$.

In Fig. 4a, each pair of source and destination (S-D) want to communicate with each other via two relay nodes, which can be seen as a group. The received signals at each relay can be given as

$$
y_{R_{i}}=\sum_{k=1}^{K} h_{S_{k} R_{i}}\left(\sqrt{a_{1, k} P_{t}} x_{1, k}+\sqrt{a_{2, k} P_{t}} x_{2, k}\right)+n_{R_{i}},
$$

where $h_{S_{k} R_{i}}$ denotes the channels from the source node $k$ to the relay node $i$ for $k \in\{1, \ldots, K\}$, and $i=1,2$. $a_{1, k}$ and $a_{2, k}$ are the power allocation factors employed at source $k$ with $a_{1, k}>a_{2, k}$ and $a_{1, k}+a_{2, k}=1$. Without loss 


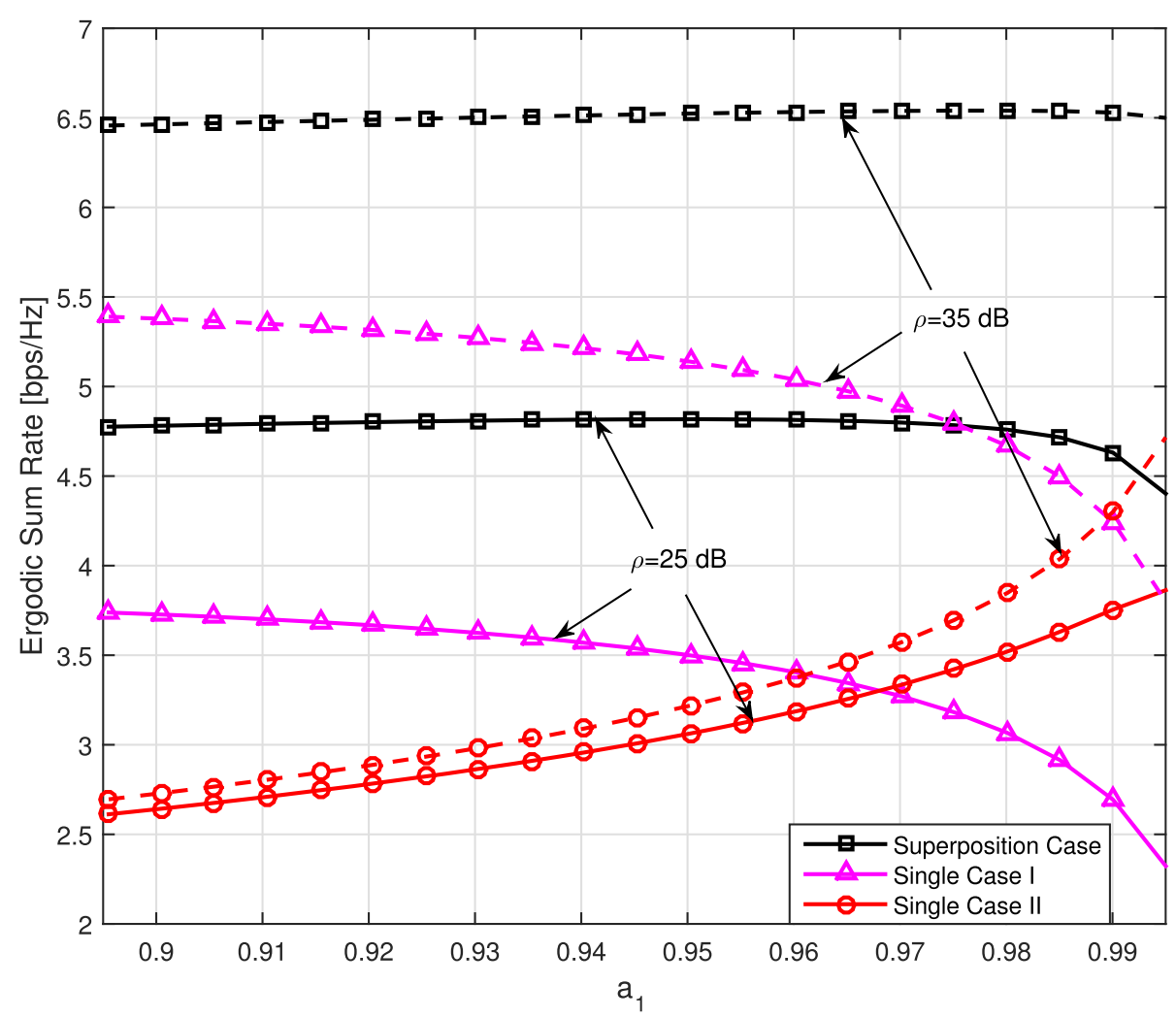

Fig. 5 The ergodic SRs achieved by our proposed and the conventional OMA schemes versus power allocation factors $a_{1}$ with fixed $b_{1}=0.35$ and $c_{1}=0.7$ for different transmit SNRs as $\rho=\{25,35\} \mathrm{dB}$

of generality, according to the NOMA principle, the symbol allocated with more transmit power will be decoded first. Similar to the proposed CTRS-NOMA superposition case, the relay will forward decoded signals with new superposition coding to the destination

$$
x_{R_{i}}=\sum_{j=1}^{K}\left(\sqrt{b_{i, 2 j-1} P_{t}} x_{1, k}+\sqrt{b_{i, 2 j} P_{t}} x_{2, k}\right),
$$

where $b_{i, j}$ is the new PA factor at relay $i$ with $\sum_{j=1}^{K}\left(b_{i, 2 j-1}+b_{i, 2 j}\right)=K$. Finally, each destination will decode their desired signal by using SIC.

In Fig. 1b, a cooperative networks consisting of one source, one destination and $L$ (even number) relay nodes is considered. It is easy to see that each two relays can be seen as a group, and the received signal for each relay is given as

$$
y_{R_{i}}=h_{S R_{i}} \sum_{m=1}^{M} \sqrt{a_{m} P_{t}} x_{m}+n_{R_{i}},
$$

where $a_{m}$ is the PA factor at the source for $m \in\{1, \ldots, M\}$ with $M \leq L$ (decodable condition) and $i=1,2$. In this system model, each relay is feasible to follow the proposed three transmission strategies. It is worth noting that the total relay power constraint should be $P_{t}$. In addition, for the CTRS-NOMA superposition case, there are total ( $T+$ 1) $M$ PA factors, while for the CTRS-NOMA single case, it is $T+M$.

Since that the finite power allocation factors and superposed transmission symbols should be considered which is quite involved to derive the closed-form expressions of the SR and PA factors, our future work will focus on finding an approximate way to solve the problems.

\section{Numerical results}

In this section, we examine the performance of our proposed CTRS-NOMA schemes in terms of the ergodic SR. All results are averaged over 80,000 channel realizations. In the following figures, we use "simulation" and "analysis" to denote the simulation and analytical results, respectively. Figures 3 and 4 depict the ergodic SR performance of our proposed CTRS-NOMA single case I, CTRS-NOMA single case II, CTRS-NOMA superposition case, and the TDMA schemes versus the transmission SNR. We have set fixed $\alpha_{S R_{1}}=5, \alpha_{S R_{2}}=1, \alpha_{R_{1} D}=2$, $\alpha_{R_{2} D}=20, b_{1}=0.4$, and $a_{1}=c_{1}=\{0.6,0.8\}$ in Fig. 3, while $a_{1}=c_{1}=0.7, b_{1}=0.3, \alpha_{S R_{1}}=\alpha_{S R_{2}}=10$, 


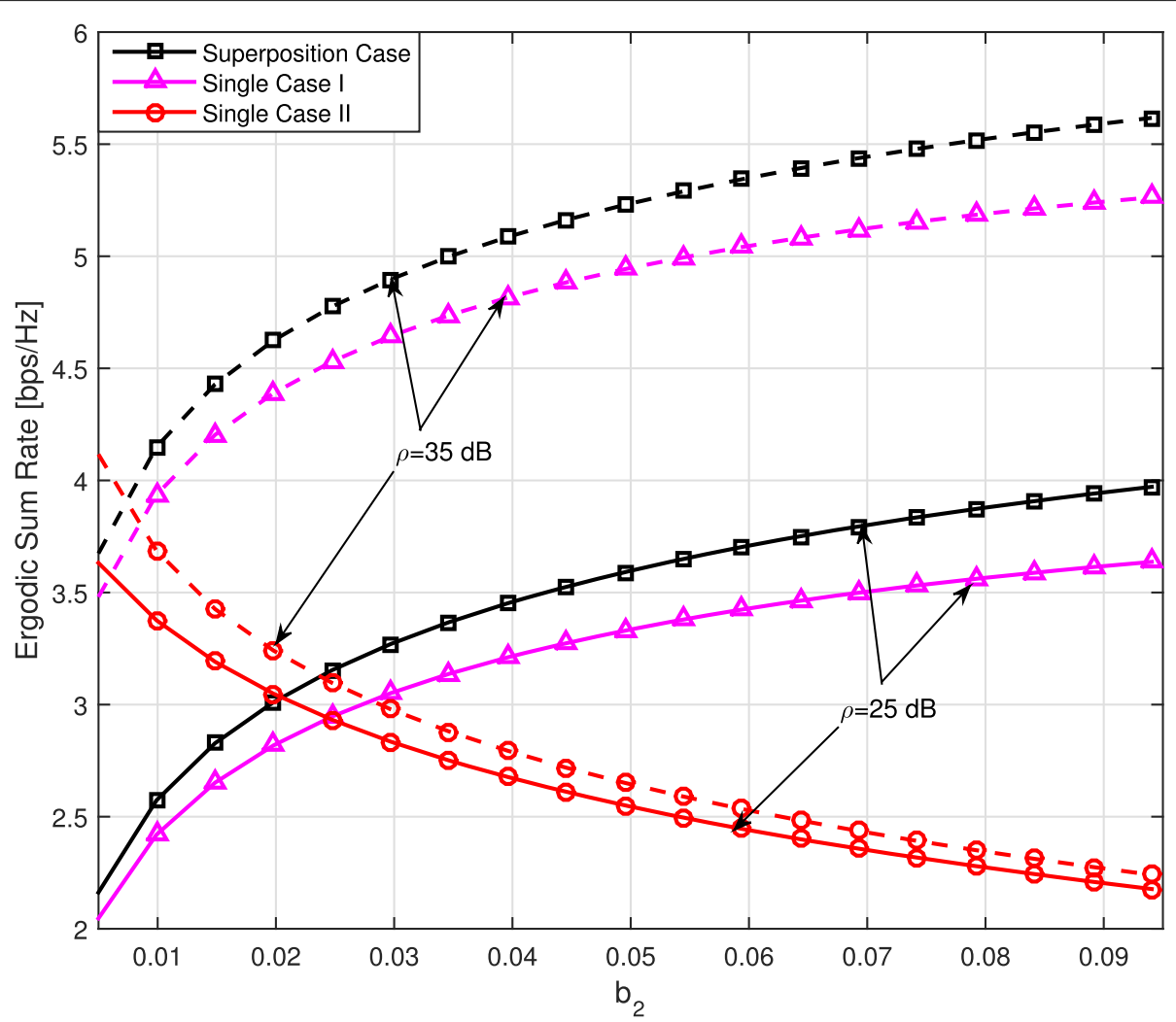

Fig. 6 The ergodic SRs achieved by our proposed CTRS-NOMA single case I, CTRS-NOMA single case II, and CTRS-NOMA superposition case versus power allocation factors $b_{2}$ for different transmit SNRs as $\rho=\{25,35\} \mathrm{dB}$

$\alpha_{R_{1} D}=2$, and $\alpha_{R_{2} D}=\{10,40\}$ in Fig. 2. It is clear that the CTRS-NOMA superposition case shows a better performance compared with other three schemes, and there is a good match between the simulation results and the analysis results, especially at the high SNR region, which supports the practical utility of our design. Remarkably, in Fig. 4, with increased PA factor $a_{1}$ and $c_{1}$, the ergodic SR performance of CTRS-NOMA single case I and CTRS-NOMA superposition case are also improved, but decreased for the CTRS-NOMA single case II. Particularly, the improvement ergodic SR of the CTRS-NOMA superposition case is small. This is because, for high SNR case, i.e., $\rho \gg 1$, the ergodic rate of $x_{1}$ is given as $C_{D}^{\left(x_{1}\right)} \sim \frac{1}{2} \log _{2}\left(1+\min \left\{\frac{a_{1}}{a_{2}}, \frac{2 b_{1}}{b_{2}}\right\}\right)=\frac{1}{2} \log _{2}\left(1+\frac{a_{1}}{a_{2}}\right)$, for $a_{1}=\{0.6,0.8\}$ and $b_{1}=0.4$. Onthe other hand, in Fig. 4 , the ergodic SR performance of CTRS-NOMA single case II and CTRS-NOMA superposition case have improved along with the increased $\alpha_{R_{2} D}$ while no meaningful change can be observed for the for the CTRS-NOMA single case I.

Figures 5, 6, and 7 depict the ergodic SR performance with respect to power allocation factors $a_{1}$ and $b_{2}$ for the CTRS-NOMA superposition case, CTRS-NOMA single case I, and CTRS-NOMA single case II, respectively. For both figures, We have set fixed $\alpha_{S R_{1}}=\alpha_{S R_{2}}=10, \alpha_{R_{1} D}=$ $\alpha_{R_{2} D}=2$. In Fig. 5, comparisons are made with fixed $b_{1}=0.35$ and $c_{1}=0.7$ for different transmit SNRs as $\rho=\{25,35\} \mathrm{dB}$. As seen from the figure, there exists an optimal value of $a_{1}$ that maximizes the ergodic SR for the CTRS-NOMA superposition case and CTRS-NOMA single case I, which always outperforms the maximum value of the CTRS-NOMA single case II. In addition, with the increase of the SNR, the corresponding $a_{1}$ for the optimal ergodic SR will be close to 1. Particularly, in Figs. 6 and 7 , to simplify the analysis, we further assume that $c_{2}=2 * b_{2}$. From the Figs. 6 and 7, we observe that the optimal SR exists when $b_{2}$ is close to 0.5 for the CTRSNOMA superposition case; and $b_{2}$ is close to 0 for the other two cases. Furthermore, for an increased $a_{1}$, the corresponding ergodic SR will be also increased for these three cases.

Figures 8,9 , and 10 compare the ergodic SR performance of the proposed CTRS-NOMA schemes versus power allocation factors with fixed $\alpha_{S R_{1}}=1, \alpha_{S R_{2}}=10$, $\alpha_{R_{1} D}=1, \alpha_{R_{2} D}=2$, and $\rho=25 \mathrm{~dB}$. The maximum results of the ergodic SR are $3.9059 \mathrm{bps} / \mathrm{Hz}, 3.2252 \mathrm{bps} / \mathrm{Hz}$, and $3.3605 \mathrm{bps} / \mathrm{Hz}$, respectively, for Figs. 6, 7, and 8. In sum, 


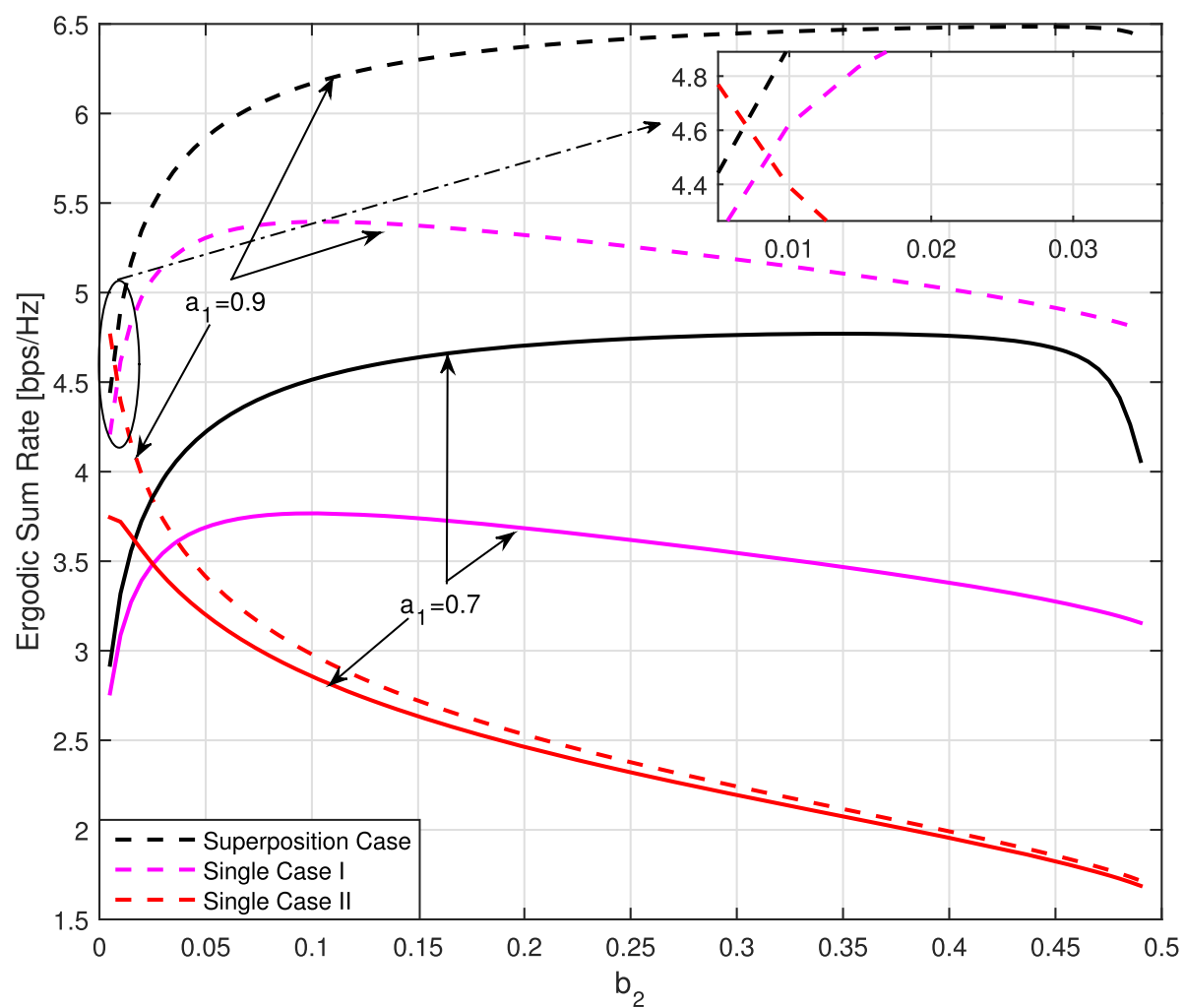

Fig. 7 The ergodic SRs achieved by our proposed CTRS-NOMA single case I, CTRS-NOMA single case II, and CTRS-NOMA superposition case versus power allocation factors $b_{2}$

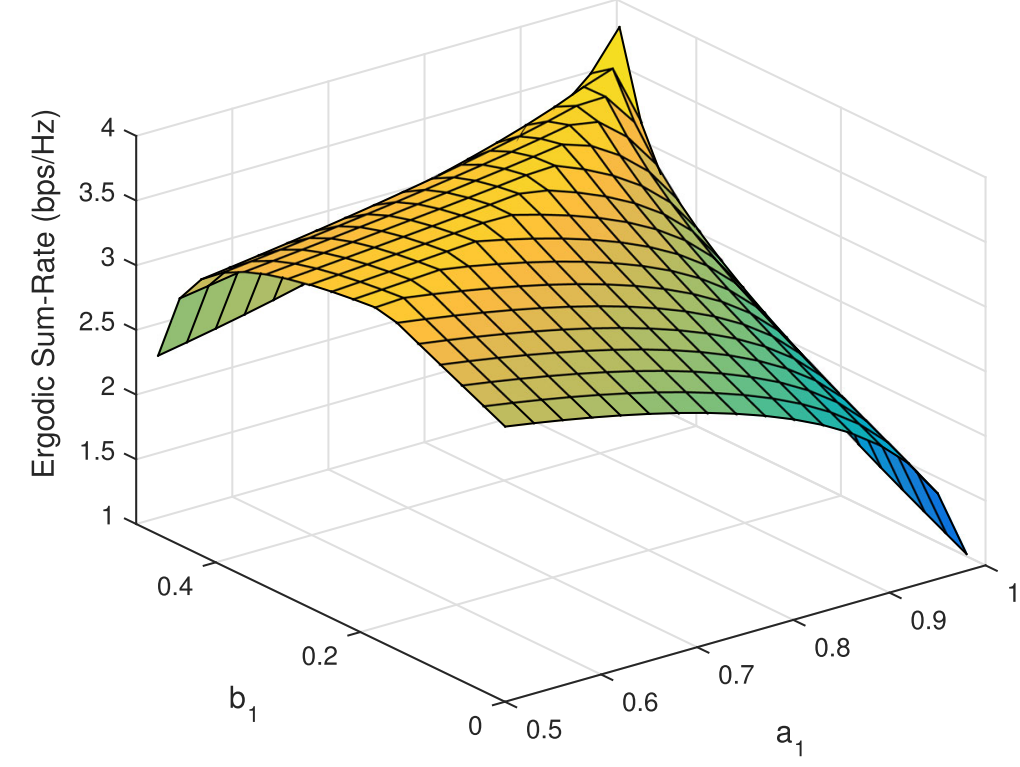

Fig. 8 The ergodic SRs achieved by our proposed CTRS-NOMA superposition case versus power allocation factors $a_{1}$ and $b_{1}$ with fixed $\alpha_{S R_{1}}=1$, $\alpha_{S R_{2}}=10, \alpha_{R_{1} D}=1, \alpha_{R_{2} D}=2$, and $\rho=25 \mathrm{~dB}$ 


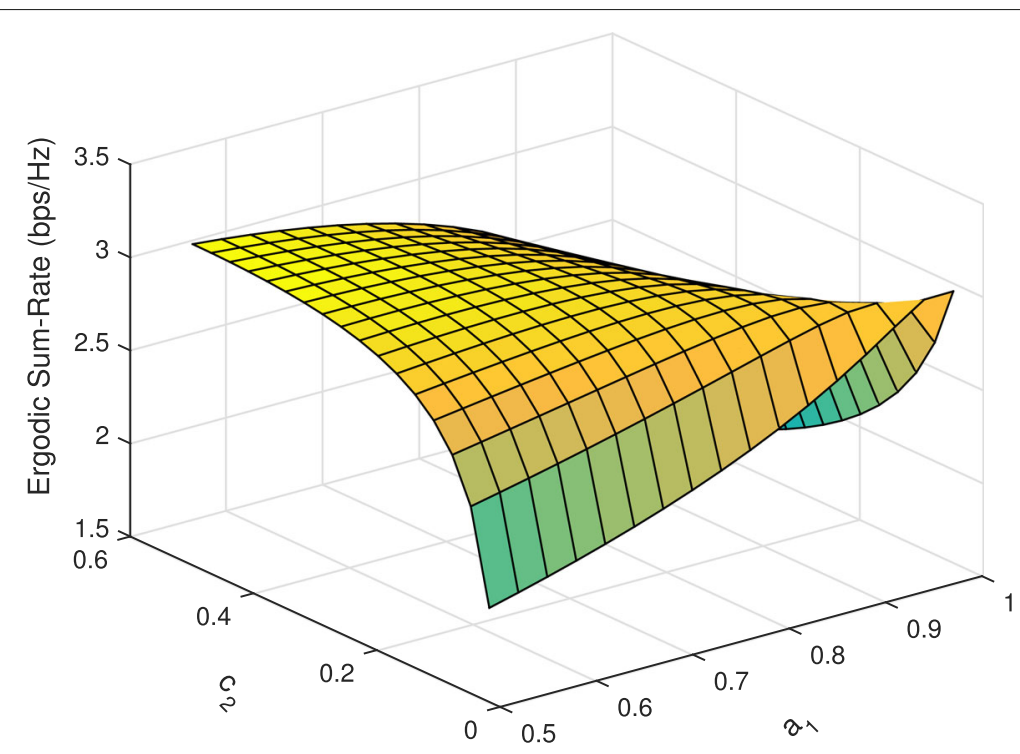

Fig. 9 The ergodic SRs achieved by our proposed CTRS-NOMA single case I versus power allocation factors $a_{1}$ and $c_{2}$ with fixed $\alpha_{S R_{1}}=1$, $\alpha_{S R_{2}}=10, \alpha_{R_{1} D}=1, \alpha_{R_{2} D}=2$, and $\rho=25 \mathrm{~dB}$

the ergodic SR of CTRS-NOMA superposition case has an outstanding advantage over the other two CTRS-NOMA schemes.

\section{Conclusions}

In this paper, considering the CTRS-NOMA system, two cases named CTRS-NOMA superposition case and CTRS-NOMA single case have been studied. Specifically, for the CTRS-NOMA single case, two PA strategies have been investigated. Also, for each one of three PA schemes, the closed-form expression for the achievable ergodic SR is derived. Numerical results have been presented to corroborate the theoretical analyses, and the results have shown us that the performance of the ergodic SR for the CTRS-NOMA superposition case gains a significant improvement and also outperforms the CTRS-NOMA single case. It remains a future work to investigate the design rule for the time slot length and the efficient slot assignment method for users with different QoS requirements.

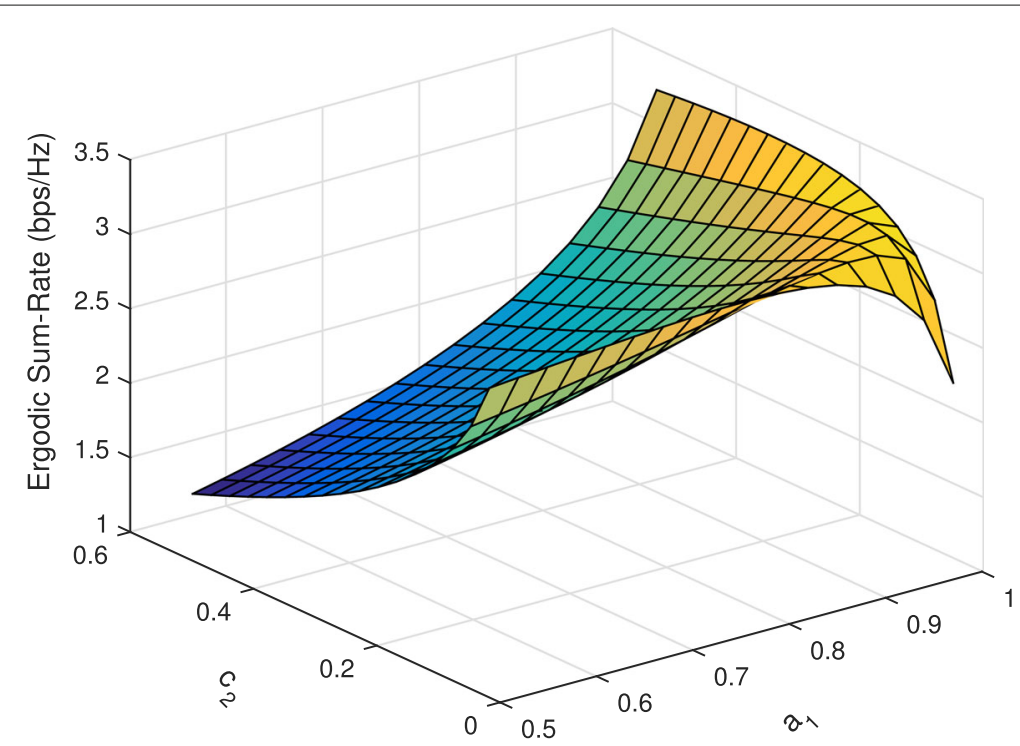

Fig. 10 The ergodic SRs achieved by our proposed CTRS-NOMA single case II versus power allocation factors $a_{1}$ and $c_{2}$ with fixed $\alpha_{S R_{1}}=1$, $\alpha_{S R_{2}}=10, \alpha_{R_{1} D}=1, \alpha_{R_{2} D}=2$, and $\rho=25 \mathrm{~dB}$ 


\begin{abstract}
Abbreviations
AF: Amplify-and-forward; AWGN: Additive white Gaussian noise CDRT: Coordinated direct and relay transmission; CRN: Cooperative relay networks; CSI: Channel state information; DF: Decode-and-forward; FDMA: Frequency-division multiple access; MRC: Maximum ratio combining; NOMA: Non-orthogonal multiple access; OFDM: Orthogonal frequency division multiplexing; OMA: Orthogonal multiple access: SIC: Successive interference cancellation; SR: Sum-rate; PA: Power allocation; SWIPT: Simultaneous wireless information and power transfer; TDMA: Time-division multiple access;
\end{abstract}

\section{Acknowledgements}

The authors would gratefully acknowledge the grants from the National Natural Science Foundation of China (61371113, 61401241, 61501264, 61571315 and 61631004), and the Nantong University-Nantong Joint Research Center for Intelligent Information Technology (KFKT2017B01).

Finally, we would like to thank the Editor and Reviewer for their constructive remarks and careful reading of our paper, which were essential in improving the overall presentation of the paper.

\section{Availability of data and materials}

The authors declare that all the data and materials in this manuscript are available.

\section{Authors' contributions}

$W D, J H$, and $G Z$ conceived and designed the study. WD, YJ, and QS performed the simulations. WD and JH wrote the paper. JH, GZ, JC, YJ, and QS reviewed and edited the manuscript. All authors read and approved the manuscript.

\section{Competing interests}

The authors declare that they have no competing interests.

\section{Publisher's Note}

Springer Nature remains neutral with regard to jurisdictional claims in published maps and institutional affiliations.

\section{Author details}

${ }^{1}$ School of Electronics and Information, Nantong University, Nantong, China.

${ }^{2}$ School of Electronics and Information, Soochow University, Suzhou, China.

${ }^{3}$ Division of Electronic and Information Engineering, Chonbuk National University, Jeonju, South Korea. ${ }^{4}$ Nantong Research Institute for Advanced Communication Technologies, Nantong 226019, Jiangsu, China.

\section{Received: 4 January 2018 Accepted: 4 June 2018}

Published online: 28 June 2018

\section{References}

1. Z Ding, M Peng, HV Poor, Cooperative non-orthogonal multiple access in 5G systems. IEEE Commun. Lett. 19(8), 1462-1465 (2015)

2. Y Zhang, H Wang, T Zheng, Q Yang, Energy-efficient transmission in non-orthogonal multiple access. IEEE Tans. Veh. Technol. 66(3), 2852-2857 (2017)

3. Y Wang, W Feng, L Xiao, Y Zhao, S Zhou, Coordinated multi-cell transmission for distributed antenna systems with partial CSIT. IEEE Commun. Lett. 16(7), 1044-1047 (2012)

4. W Feng, Y Wang, N Ge, J Lu, J Zhang, Virtual MIMO in multi-cell distributed antenna systems: coordinated transmissions with large-scale CSIT. IEEE J. Sel. Areas Commun. 31(10), 2067-2081 (2013)

5. Z Ding, F Adachi, HV Poor, The application of MIMO to non-orthogonal multiple access. IEEE Trans. Wirel. Commun. 15(1), 537-552 (2016)

6. Z Ding, X Lei, GK Karagiannidis, R Schober, J Yuan, VK Bhargava, A survey on non-orthogonal multiple access for $5 \mathrm{G}$ networks: research challenges and future trends. IEEE J. Sel. Areas Commun. 35(10), 2181-2195 (2017)

7. SM Riazul Islam, N Avazov, OA Dobre, K Kwak, Power-domain non-orthogonal multiple access (NOMA) in 5G systems: Potentials and Challenges. IEEE Commun. Surv. Tutor. 19(2), 721-742 (2016)

8. SM Riazul Islam, M Zeng, OA Dobre, K Kwak, Resource allocation for downlink NOMA systems: Key techniques and open issues. IEEE Wirel. Commun. Mag. 25(2), 40-47 (2018)

9. F Md. Kader, MB Shahab, SY Shin, Exploiting non-orthogonal multiple access in cooperative relay sharing. IEEE Commun. Lett. 21(5), 1159-1162 (2017)
10. B He, A Liu, N Yang, VKN Lau, On the design of secure non-orthogonal multiple access systems. IEEE J. Sel. Areas Commun. 35(10), 2196-2206 (2017)

11. Y Liu, Z Qin, M Elkashlan, Y Gao, L Hanzo, Enhancing the physical layer security of non-orthogonal multiple access in large-scale networks. IEEE Trans. Wirel. Commun. 16(3), 1656-1672 (2017)

12. Q Yang, H-M Wang, D Kwan Ng, M Lee, NOMA in downlink SDMA with limited feedback: performance analysis and optimization. IEEE J. Sel. Areas Commun. 35(10), 2281-2294 (2017)

13. $Y$ Zhang, H-M Wang, Q Yang, Z Ding, Secrecy sum rate maximization in non-orthogonal multiple access. IEEE Commun. Lett. 20(5), 930-933 (2016)

14. C Chen, W Cai, X Cheng, L Yang, Y Jin, Low complexity beamforming and user selection schemes for 5 G MIMO-NOMA systems. IEEE J. Sel. Areas Commun. 35(12), 2708-2722 (2017)

15. NT Do, D Costa, TQ Duong, B An, A BNBF user selection scheme for NOMA-based cooperative relaying systems with SWIPT. IEEE Commun. Lett. 21(3), 664-667 (2017)

16. Z Yang, Z Ding, P Fan, N Al-Dhahir, The impact of power allocation on cooperative non-orthogonal multiple access networks with SWIPT. IEEE Trans. Wirel. Commun. 16(7), 4332-4343 (2017)

17. Y Xu, C Shen, Z Ding, X Sun, S Yan, G Zhu, Z Zhong, Joint beamforming and power-splitting control in downlink cooperative SWIPT NOMA systems. IEEE Trans. Signal Process. 65(18), 4874-4886 (2017)

18. Y Liu, Z Ding, M Elkashlan, HV Poor, Cooperative non-orthogonal multiple access with simultaneous wireless information and power transfer. IEEE J. Sel. Areas Commun. 34(4), 938-953 (2016)

19. J Choi, Power allocation for max-sum rate and max-min rate proportional fairness in NOMA. IEEE Commun. Lett. 20(10), 2055-2058 (2016)

20. M Hojeij, CA Nour, J Farah, C Douillard, Waterfilling-based proportional fairness scheduler for downlink non-orthogonal multiple access. IEEE Commun. Lett. 6(2), 230-233 (2017)

21. M Wen, E Basar, Q Li, B Zheng, M Zhang, Multiple-mode orthogonal frequency division multiplexing with index modulation. IEEE Trans. Commun. 65(9), 3892-3906 (2017)

22. M Wen, B Ye, E Basar, Q Li, F Ji, Enhanced orthogonal frequency division multiplexing with index modulation. IEEE Trans. Wirel. Commun. 16(7), 4786-4801 (2017)

23. J Li, X Jiang, Y Yan, W Yu, S Song, MH Lee, Low complexity detection for quadrature spatial modulation systems. Wirel Pers. Commun. 95(4), 4171-4183 (2017)

24. M Wen, X Cheng, M Ma, B Jiao, HV Poor, On the achievable rate of OFDM with index modulation. IEEE Trans. Signal Process. 64(8), 1919-1932 (2016)

25. J Li, M Wen, X Jiang, W Duan, Space-time multiple-mode orthogonal frequency division multiplexing with index modulation. IEEE Access. 5 23212-23222 (2017)

26. J Li, M Wen, X Cheng, Y Yan, S Song, MH Lee, Generalised pre-coding aided quadrature spatial modulation. IEEE Trans. Veh. Technol. 66(2), 1881-1886 (2016)

27. X Zhu, Z Wang, J Cao, NOMA-based spatial modulation. IEEE Access. 5, 3790-3800 (2017)

28. Y Chen, L Wang, Y Ai, B Jiao, L Hanzo, Performance analysis of NOMA-SM in vehicle-to-vehicle massive MIMO channels. IEEE J. Sel. Areas Commun. 35(12), 2653-2666 (2017)

29. H Lin, F Gao, S Jin, G Ye Li, A new view of multi-user hybrid massive MIMO Non-orthogonal angle division multiple access. IEEE J. Sel. Areas Commun. 35(10), 2268-2280 (2017)

30. $\mathrm{HXie}, \mathrm{F}$ Gao, $\mathrm{S}$ Jin, An overview of low-rank channel estimation for massive MIMO systems. IEEE Access. 4(99), 7313-7321 (2016)

31. HXie, F Gao, S Zhang, S Jin, A united transmission strategy for TDD/FDD massive MIMO systems with spatial basis expansion Model. IEEE Trans. Veh. Technol. 66(4), 3170-3184 (2017)

32. Y Li, M Jiang, Q Zhang, Q Li, J Qin, Cooperative non-orthogonal multiple access in multiple-input-multiple-output channels. IEEE Trans. Wirel. Commun. 17(3), 2068-2079 (2018). http://ieeexplore.ieee.org/document/ 8247238/

33. Q Zhang, Q Li, J Qin, Robust beamforming for non-orthogonal multiple access systems in MISO channels. IEEE Trans. Veh. Technol. 65(2), 10231-10236 (2016) 
34. C Xue, Q Zhang, Q Li, J Qin, Joint power allocation and relay beamforming in nonorthogonal multiple access amplify-and-forward relay networks. IEEE Trans. Veh. Technol. 66(8), 7558-7562 (2017)

35. R Jiao, L Dai, J Zhang, R MacKenzie, M Hao, On the performance of NOMA-based cooperative relaying systems over Rician fading channels. IEEE Trans. Veh. Technol. 66(12), 11409-11413 (2017)

36. $X$ Liang, Y Wu, DW Ng, Y Zuo, S Jin, H Zhu, Outage performance for cooperative NOMA transmission with an AF relay. IEEE Commun. Lett. 21(11), 2428-2431 (2017)

37. S Zhang, B Di, L Song, Y Li, Sub-channel and power allocation for non-orthogonal multiple access relay networks with amplify-and-forward protocol. IEEE Trans. Wirel. Commun. 16(4), 2249-2261 (2017)

38. J Men, J Ge, Non-orthogonal multiple access for multiple antenna relaying networks. IEEE Commun. Lett. 19(10), 1686-1689 (2015)

39. JB Kim, IH Lee, Capacity analysis of cooperative relaying systems using non-orthogonal multiple access. IEEE Commun. Lett. 19(11), 1949-1952 (2015)

40. M Xu, M Wen, F Ji, W Duan, Novel receiver design for the cooperative relaying system with non-orthogonal multiple access. IEEE Commun. Lett. 20(8), 1679-1682 (2016)

41. W Duan, M Wen, Z Xiong, M Lee, Two-stage power allocation for dual-hop relaying systems with non-orthogonal multiple access. IEEE Access. 5, 2254-2261 (2017)

42. IS Gradshteyn, IM Ryzhik, Table of integrals, series, and products, 7thed. (Academic Press, Manhattan, 2007)

\section{Submit your manuscript to a SpringerOpen ${ }^{\circ}$ journal and benefit from:}

- Convenient online submission

Rigorous peer review

- Open access: articles freely available online

- High visibility within the field

- Retaining the copyright to your article

Submit your next manuscript at $>$ springeropen.com 